\title{
Retinal detachment in AIDS: long term results after repair with silicone oil
}

\author{
A R Irvine, L Lonn, D Schwartz, M Zarbin, F Ballesteros, S Kroll
}

\begin{abstract}
Aims-To study the long term results of vitrectomy and silicone oil injection in AIDS patients with retinal detachment due to viral retinitis.

Methods-A consecutive series of 83 eyes in 75 AIDS patients who were treated with vitrectomy and silicone oil for retinal detachment due to viral retinitis was studied prospectively and followed until the last patient in the series had died.

Results-Median postoperative survival was 6 months, but $20 \%$ of patients survived 12 months or more. Whereas best corrected visual acuity was $20 / 100$ or better in over half the patients 1-2 months after operation, there was a distressing decrease in acuity by 6 months, owing to a variety of factors. Patients operated on before macular detachment did not have significantly better postoperative vision than those operated within a week after macular detachment. Almost all patients who had already lost vision in the fellow eye and many who had cytomegalovirus retinitis in the fellow eye with retention of good vision had their quality of life improved by the surgery in that the operated eye eventually became the better seeing eye. In no patient whose fellow eye was normal and free of retinitis, however, did the operated eye ever become the better eye. macula detaches and that patients whose fellow eye is free of retinitis with normal vision are unlikely to have their quality of life improved significantly by the surgery. (Br f Ophthalmol 1997;81:180-183)
\end{abstract}

Department of

Beckman Vision

Center, University of

California, San

Francisco, USA

A R Irvine

L Lonn

D Schwartz

M Zarbin

F Ballesteros

S Kroll

Correspondence to:

Alexander R Irvine, $\mathrm{MD}$,

Department of

Ophthalmology, Beckman

Vision Center, 10 Kirkham

Street, University of

California, San Francisco,

CA 94143-0730, USA.

Accepted for publication 4 November 1996

The use of vitrectomy and silicone oil to repair retinal detachment due to viral retinitis in AIDS patients was introduced by Freeman et $a l^{1}$ and has become generally accepted. ${ }^{2-11}$
Reports have differed, however, regarding the visual results. Initial problems with early postoperative visual loss have improved with changes in technique. ${ }^{8}$ These have included the recognition that these patients are unusually susceptible to central retinal artery occlusion by increased intraocular pressure, so that the height of the infusion bottle during surgery and the pressures induced during and immediately after silicone oil injection are kept relatively low. ${ }^{3}$ Also important has been the use of a laser barrier and/or more complete silicone fill to prevent spread of recurrent inferior detachment into the fovea. ${ }^{6}$

While the problem of immediate or early postoperative visual loss has diminished, the question of later visual loss remains. A recent large, multicentred trial of the efficacy of silicone oil in AIDS detachments looked only at anatomic reattachment and the retention of 'ambulatory vision', and did not address lesser changes in visual acuity during the postoperative course. ${ }^{12}$ Those reported series which have followed their patients until death, rather than including patients still alive and only a few months after operation and which have followed the course of visual acuity, have been relatively small. They have, however, frequently shown that after reaching rather good 'best postoperative acuity' at 1 to 2 months, their patients had a discouraging later drop in acuity, after 3 to 6 months. ${ }^{3411}$ This has seemed in excess of what could be accounted for by cataract or obvious macular pathology and has led some to question whether there is progressive optic atrophy due either to the cytomegalovirus (CMV) infection or to possible silicone oil retinal toxicity. ${ }^{11}$ This question of late visual loss must be included in any evaluation of the indications for this surgery. The present series of patients is the largest yet reported when all patients have been followed until death.

\section{Materials and methods}

Eighty three consecutive eyes of 75 AIDS patients who underwent vitrectomy and intravitreal silicone oil injection for detachment due to viral retinitis between October 1989 and December 1993 were evaluated. At the time of statistical analysis the last of these patients had died. The patients were part of a prospective 
study on the efficacy of silicone oil in AIDS patients. The study was approved by our local institutional review board as well as by the FDA, and all patients were adults who had signed an informed consent document. A standard three port pars plana vitrectomy was performed, in which we tried to peel the posterior hyaloid away from the healthy retina if it was not spontaneously detached, but not peeling hyaloid nor membranes from atrophic retina if they did not come away spontaneously. The retina was reattached by endodrainage and air-fluid exchange, and in most cases, endolaser was used to create a barrier below the inferior temporal arcade and coming up above the horizontal meridian nasal to the optic nerve and temporal to the macula, to prevent postoperative recurrent inferior detachment from coming into the macula. A silicone fill was achieved, as completely as possible, by slowly releasing the air through a 27 gauge needle placed through the most superior pars plana while the eye was rotated downwards and the silicone was injected through the inferior cannula. The patients were kept in strict face down position for the first night after operation. Six of the eyes operated on in the early portion of this series underwent encircling scleral buckling at the time of vitrectomy and silicone injection.

Postoperative follow up was often difficult owing to the severe illness of these patients. Whenever possible, they were seen in the surgeon's office, but data were also obtained from referring physicians, from hospital bedside visits, and occasionally from the patients' friends and family. In four eyes of three patients, we failed to obtain a postoperative visual acuity. These were very ill patients who presented only when their second eye detached. A total of 397 post-treatment visual acuity measurements were taken.

For statistical analysis, the time to onset of significant cataract was estimated by the Kaplan-Meier ${ }^{13}$ product limit method. The effects of covariates (age, pretreatment refraction, macular status, preoperative visual acuity, and degree of retinal detachment) on the time to visual decrease were examined using multivariate Cox models. ${ }^{14}$ The effect of macular status on visual outcome was also examined by non-parametric Mann-Whitney tests at various follow up times. The probability at all postoperative times of having visual acuity at least as good as a given threshold (20/50, $20 / 100,20 / 200,20 / 400$ ) were estimated nonparametrically by counting process methods. ${ }^{15}$ These estimates were then smoothed for visual presentation. All statistical analyses were performed with S-PLUS for Windows (Mathsoft, Inc, Seattle, WA, USA) supplemented with user written macros.

\section{Results}

SURVIVAL

The median survival was 6 months, but the distribution had a wide spread. Twenty five per cent of the patients died within 2 months of surgery while $20 \%$ lived 12 months or more

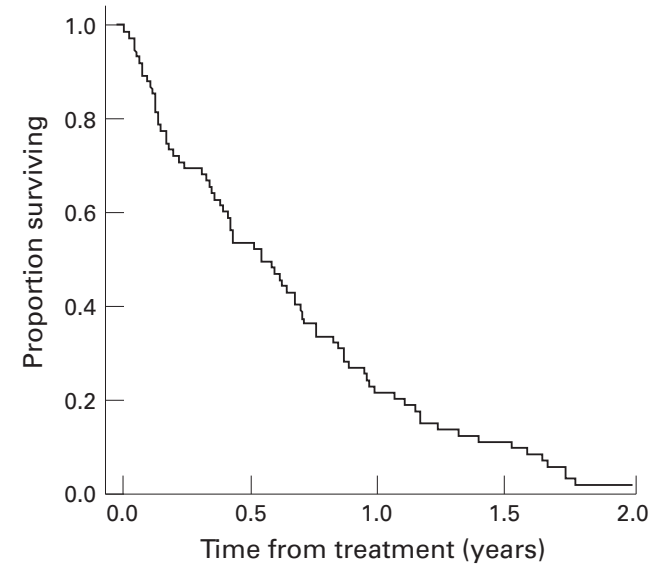

Figure 1 Empirical survival function of the proportion of patients surviving from the date of treatment. The time patients surviving from the date of treatment. The time cases with bilateral treatment. All cases were followed until the time of death. Percentage survival times-0.0-0.5 year $47 \%$; $0.5-1.0$ year 32\%; more than 1.0 year $21 \%$.

after operation (Fig 1). For purposes of calculating survival, in those eight patients who underwent bilateral surgery, we utilised only the time from operation on the first eye.

VISION

In $81 \%$ of the eyes the macula was detached at the time of surgery and, in a few cases, retinitis involved the macula before the detachment. Of the 79 eyes for which postoperative acuities were obtained, $32 \%$ of patients achieved a best postoperative vision of $20 / 20-20 / 50,34 \%$ achieved 20/60-20/100, 22\% reached 20/200$20 / 400$, and only $13 \%$ achieved less than $20 / 400$. This best acuity was usually achieved by 1-2 months after operation. The disappointing finding was how rapidly the acuity dropped thereafter. By 6 months, the numbers of eyes in the 20/20-20/50 and the 20/60-20/ 100 range had been cut by about half (Fig 2). There were a number of causes for the decrease in acuity. Of 30 eyes which had a best postoperative vision better than or equal to 20/100 and which were followed 6 months, only nine maintained vision in the $20 / 100$ or better range at 6 months. In six eyes, spread of retinitis into the fovea explained the decrease in vision, and in two eyes recurrent retinal detachment crossed into the fovea from below. In another six eyes the vision of $20 / 200$ or worse was felt to be consistent with the cataract. In the remaining seven eyes no cause other than optic atrophy was recognised.

The macula was detached in 67 eyes and attached in 16 eyes at the time of surgery. Like Kupperman et al, we found that postoperative vision was not significantly affected by whether the macula was detached preoperatively. ${ }^{6}$ Almost all macula off cases were operated on within 4 days of the time the macula detached. Like Freeman et $a l,{ }^{5}$ we have been surprised by the rapid and excellent return in acuity in patients with CMV detachments whose macula had been off for up to a week. Freeman et al suggested that the youth and relatively solid vitreous in these patients may have kept 


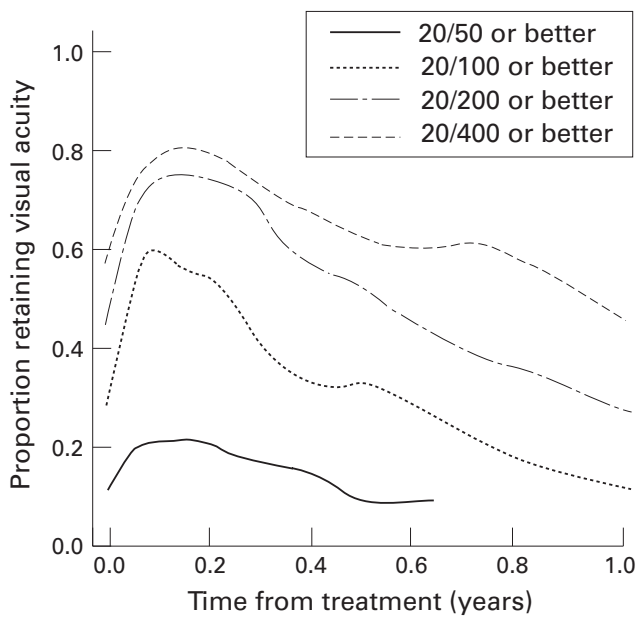

Figure 2 Non-parametric smoothed estimates for the proportion of eyes retaining visual acuity of at least 20/50, $20 / 100,20 / 200$, and 20/400. The proportion of time 0 is equal to the cohort proportion at the time of treatment. The post-treatment improvement in visual acuity decreased to the pretreatment level at about 6 months.

the macular detachments shallow and this accounted for the rapid visual return.

A postoperative refraction was recorded in 52 of the 77 eyes which did not undergo scleral buckling at the time of vitrectomy. The change from preoperative to first postoperative refraction was relatively uniform with the great majority showing between a 5 and 7 dioptre hyperopic shift and a mean change of 6.1 dioptres. Preoperative refraction was taken from the patients' spectacle prescription before the detachment in cases with macular detachment.

The mean time from onset of CMV retinitis until retinal detachment surgery was 5.5 months for the eyes with greater than 3 dioptres of myopia, 6.5 months for those with 1-3 dioptres of myopia, and 8 months for those with less than 1 dioptre of myopia, supporting the concept that myopia may be a predisposing factor to detachment in these patients. ${ }^{216}$

Cataract seems an inevitable complication of vitrectomy and complete silicone fill. We graded cataracts semiquantitatively on a $1-4$ scale of severity. A grade 3 cataract was defined as that degree of lens opacity which the observer felt might reduce vision to 20/200. The time of onset of a grade 3 cataract is shown in Figure 3, with a median time of onset of 7 months.

In determining outcome, the most important factor is whether the patient's quality of life was helped by the surgery. The most important index in this regard was whether the operated eye became the patient's better eye. In those cases where the fellow eye had already lost useful vision, 21 of 23 operated eyes (91\%) obtained useful vision and were the better eye postoperatively. In those cases where the fellow eye had viral retinitis but maintained good foveal vision at the time of surgery, 15 of 35 $(43 \%)$ operated eyes eventually became the better eye at least for some period of time. This includes those eight cases where the second eye later detached. In those cases where the fellow eye had normal foveal vision and no retinitis, 0

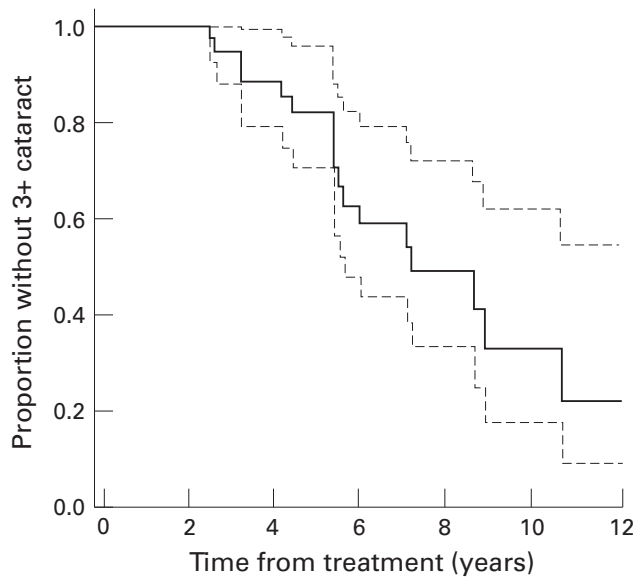

Figure 3 Kaplan-Meier estimate of the proportion of patients developing 3+ nuclear cataracts. Patients without known cataract development were censored at their last ophthalmic visit. The first follow up visit with $3+$ cataract was used for the time of onset. The $95 \%$ confidence intervals (broken lines) are also displayed. The estimated median time to 3+ cataract was 7.3 months.

of $25(0 \%)$ operated eyes later became the better eye.

\section{Discussion}

Since survival was 6 months or more in half the cases in this series, and 12 months or more in $20 \%$, it seems important to look past the 'best postoperative acuity'. We found a discouraging decline in vision after the best acuity was reached at 1-2 months. Even the initial best results, however, are a bit misleading, for the silicone oil neutralises the back surface of the lens, thus producing an approximately 6 dioptre hyperopic shift. ${ }^{17}{ }^{18}$ If the other eye still sees normally, this produces such anisometropia that the operated eye is usually not corrected. The patient thus gets little benefit from the operated eye as long as the other eye maintains normal vision. Since cataract appears to be one major cause of postoperative visual loss, one would expect a cataract extraction with intraocular lens implantation (using either a meniscus or a convex plano IOL) ${ }^{19}$ would allow restoration of both visual acuity and refractive balance between the two eyes. Unfortunately, in the patients we have seen who have undergone cataract surgery, both from this series and those done more recently, none has achieved better than 20/200 following cataract removal.

The poor visual results after cataract surgery and the patients with visual loss with no obvious cause other than optic atrophy, raise the question of whether CMV retinitis can cause an indolent progressive optic atrophy. ${ }^{11}$ Alternatively the question of possible silicone oil retinal toxicity arises. ${ }^{11}$ To study the question of postoperative optic atrophy, Kupperman et al graded the optic atrophy photographically and found no difference on masked comparison with the fellow eye. ${ }^{6}$ Any photographic evaluation of disc pallor comparing one eye with postoperative nuclear sclerosis and the fellow eye with a clear lens seems questionable, however. The only long term clinical studies on silicone oil have involved patients 
with proliferative vitreoretinopathy. ${ }^{20}{ }^{21}$ In those series, very few patients have had best postoperative vision better than $20 / 200$, and some authors have stated that the poor vision and complex clinical courses would have made it impossible to detect evidence of retinal toxicity. ${ }^{22}$ Thus, we have no data on a significant number of patients with vitrectomy and complete silicone fill who had early postoperative vision better than $20 / 200$ and were then followed over 6-12 months without complicating factors. We must, therefore, at least consider the possibility that silicone oil toxicity could be contributing to the optic atrophy and slow decrease in acuity seen in many of our CMV patients after 3-4 months. ${ }^{22}$

Although the present series did not have the striking preponderance of high myopes described in a much smaller, earlier group, there was a tendency for myopes to develop detachment sooner after the onset of CMV retinitis than non-myopes. In these relatively young patients, this may be a result of the earlier liquefaction of vitreous in these myopes. ${ }^{216}$ It may be an indication that high myopes with CMV retinitis need to be examined more frequently for detachment.

A major goal of any study of surgical results should be to offer guidelines on when to perform the surgery. The standard indication for surgery is that it improves the patient's quality of life. In our series, vision and thus quality of life were certainly improved when the fellow eye had already been lost, and the detachment to be operated on was in the only eye. Even when the fellow eye had good vision but had retinitis, the operated eye became the better eye, and thus improved the quality of life, in a sufficient number to warrant surgery. When the fellow eye was normal with no retinitis, however, the odds of the operated eye ever becoming the better eye were so insignificant that one must question the value of surgery. It is a psychological blow to go blind in one eye, and one might argue that patients with this terrible disease already suffer enough psychological trauma. None the less, the results of this series indicate that one should at least consider leaving the first eye unoperated if there is no retinitis in the normal fellow eye.

Another guideline the authors have taken from the results of this study has been to delay surgery usually until the macula detaches. Our reasons are multiple. A few patients have had catastrophic visual loss immediately postoperatively. Even successful cases have visual compromise due to the refractive changes, and thus note a decrease in vision postoperatively if the macula was attached before surgery. We found no significant difference in postoperative vision between patients whose macula was attached and those whose macula was detached for a few days. Finally, even the remote possibility of silicone toxicity causes us to prefer to delay surgery until the macula detaches so as to decrease the time silicone will be in contact with the retina in these patients with limited lifespan.

This work was supported in part by an unrestricted Research to Prevent Blindness Grant and NEI core grant no EY02162.

1 Freeman WR, Henderly DE, Wan WL, Causey D, Trousdale M, Green RL, et al. Prevalence, pathophysiology, and treatment of rhegmatogenous retinal detachment in treated cytomegalovirus retinitis. Am $\mathcal{f}$ Ophthalmol 987;103:527-36.

2 Irvine AR. The treatment of retinal detachment due to CMV retinitis in AIDS. Trans Am Ophthalmol Soc 1991;89: 349-67.

3 Dugel PU, Liggett PE, Lee MB, Ziogas A, Forster DJ, Smith $\mathrm{RE}$, et al. Repair of retinal detachment caused by cytomegalovirus retinitis in patients with the acquired 235-42.

4 Sidikaro Y, Silver L, Holland GN, Krieger AE. Rhegmatogenous retinal detachments in patients with AIDS and necrotizing retinal infections. Ophthalmology 1991;98:12935.

5 Freeman WR, Quiceno JI, Crapotta JA, Listhaus A, Mungria D, Aguilar MF. Surgical repair of rhegmatogenous retinal detachment in immunosuppressed patients with cytomegalovirus retinitis. Ophthalmology 1991;99: 66-74.

6 Kupperman BD, Flores-Aguilar M, Quiceno JI, Capparelli EV, Levi L, Mungria D, et al. A masked prospective evaluation of outcome parameters for cytomegalovirus-related retinal detachment surgery in patients with acquired immune deficiency syndrome. Ophthalmology 1994;101: 46-55.

7 Lim JI, Enger C, Haller JA, Campochiaro PA, Meredith TA, de Bustros S, et al. Improved visual results after surgical repair of cytomegalovirus-related retinal detachments. Ophthalmology 1994;101:264-9.

8 Garcia RF, Flores-Aguilar M, Quiceno JI , Capparelli EV, Mungria D, Kupperman BD, et al. Results of rhegmatogenous retinal detachment repair in cytomegalovirus retinitis with and without scleral buckling. Ophthalmology 1995;102:236-45.

9 Nasemann JE, Mutsch A, Wiltfang R, Klauss V. Early pars plana vitrectomy without buckling procedure in cytomegalovirus retinitis-induced retinal detachment. Retina 1995; 15:111-6.

10 Regillo CD, Vander JF, Duker JS, Fischer DH, Belmont JB, Kleiner R. Repair of retinitis-related retinal detachments with silicone oil in patients with acquired immunodeficiency syndrome. Am f Ophthalmol 1992;113:21-7.

11 Dowler JGF, Towler HMA, Mitchell SM, Cooling RJ, Lightman SL. Retinal detachment and herpesvirus retinitis in patients with AIDS. Br f Ophthalmol 1995;79:575-80.

12 Davis JL, Serfass MS, Lei M, Trask DK, Azen SP. Silicone oil in repair of retinal detachments caused by necrotizing retinitis with HIV infection. Arch Ophthalmol 1995;113: 1401-9.

13 Kaplan EL, Meier P. Non-parametric estimation from incomplete observations. F Am Stat Assoc 1958;53:457-81.

14 Cox DR. Regression models and life tables (with discussion). F Roy Stat Soc B 1972;34:187-220.

15 Keiding N, Anderson PK. Nonparametric estimation of transition intensities and transition probabilities: a case study of a two-state markov process. Appl Stat 1989;38: 319-29.

16 Geier SA, Klauss V, Goebel FD. Risk factors for development of rhegmatogenous retinal detachment in patients with cytomegalovirus retinitis. (Letter to the editor) Am f Ophthalmol 1994;117:820-1.

17 Stefannson E, Tiedeman JS. Optics of the eye with air or silicone oil. Retina 1988;8:10-9.

18 Stefannson E, Anderson MM, Landers III MB, Tiedeman JS, McCuen BW. Refractive changes from the use of silicone oil in vitreous surgery. Retina 1988;8:20-3.

19 McCartney DL, Miller KM, Stark WJ, Guyton DL, Michels RG. Intraocular lens style and refraction in eyes treated RG. Intraocular lens style and refraction in eyes
with silicone oil. Arch Ophthamol 1987;105:1385-7.

20 Chan C, Okun E. The question of ocular tolerance to intraChan C, Okun E. The question of ocular tolerance to in
vitreal liquid silicone. Ophthalmology 1986;93:651-60.

21 Sell CH, McCuen BW, Landers III MB, Machemer R. Long term results of successful vitrectomy with silicone oil for advanced proliferative vitreoretinopathy. Am $\mathcal{F}$ Ophthalmol 1987;103:24-8.

22 Sebag J. Repair of retinal detachment caused by cytomegalovirus retinitis in patients with acquired immunodeficiency syndrome. (Letter to the editor) Am $\mathcal{F}$ Ophthalmol 1992;113:346-7. 